\title{
Aquatic Life Health Quality Assessment of the Bolgoda Canal and Waga Stream with respect to Selected Physico-chemical Parameters and Bioindicators
}

\author{
F.S. Idroos ${ }^{1}$ and P.M. Manage*1 \\ ${ }^{1}$ Department of Zoology, University of Sri Jayewardenepura, Nugegoda, Sri Lanka
}

Date Received: 29-05-2012 Date Accepted: 21-08-2012

\begin{abstract}
The relationship between some physico-chemical parameters and bioindicators in Bolgoda canal and Waga stream in Colombo, Sri Lanka was assessed from February to July 2010. The objective of the study was to evaluate the quality of aquatic health in the two water bodies using some physico-chemical parameters and bioindicators. Physico-chemical parameters of the Water bodies were measured. Macro invertebrates and phytoplankton were studied as biological indicators. Quantification of macro invertebrates were carried out and Pollution Tolerant Index (PTI) of Bolgoda canal and Waga stream was calculated according to the standard manual published by the United States Environmental Protection Agency (USEPA). Macro invertebrate component in the Bolgoda canal represented both moderately pollution tolerant (Nepa cinerea, Zygopteran nymphs) and pollution tolerant (Planorbella trivolvis, Promacea bridgesi, Gerris sp, Lethocerus americanus, Plea frontalis, Cerithiidae sp, Tubifex tubifex) organisms while in Waga stream only pollution sensitive organisms (Paludomous loricatus, Paludomous zeylanicus, Cylindrostesthus productus, Plecopteran nymphs, Psephenidae larvae, Aegla sp, Ephemeropteran nymphs) were reported. The PTI value of Bolgoda canal ranged from 17.00 to 19.90 where as in Waga stream was ranged from 34.00 to 39.60 indicating poor and good water quality respectively. The phytoplankton composition of both water bodies were analyzed using Shannon Wiener Diversity Index (SWDI). SWDI for phytoplankton in Bolgoda canal was ranged between $0.674 \pm 0.36$ and $1.513 \pm 1.80$ and in Waga stream was ranged from $1.89 \pm 0.72$ - 3.01 \pm 1.89 indicating low and high diversity respectively. Data analysis by Principle Component Analysis (PCA) showed that physico-chemical parameters, PTI and SWDI of the sampling locations in Bolgoda canal and Waga stream were clustered into three distinct groups according to the site selection. Regression analysis showed temperature, $\mathrm{pH}$, DO, nitrate concentration and BOD had a significant effect on the PTI value of the Bolgoda canal and Waga stream.
\end{abstract}

Key words: Physico-chemical parameters, Bioindicators, Pollution Tolerant Index (PTI), Shannon Wienner Diversity Index (SWDI), Principal Component Analysis (PCA).

\section{Introduction}

Biological monitoring or bio- monitoring, is the use of biological response to assess changes in the environment, generally changes due to anthropogenic causes (Ramakrishna, 2003) and is a valuable assessment tool that is receiving increased use in water quality monitoring programs (Kennish, 1992). Bio- monitoring involves the use of indicators, indicator species or indicator communities such as benthic

*Correspondence: path@sjp.ac.lk

Tel: +94112804515

ISSN 2235-9370 Print / ISSN 2235-9362 Online (C2012 University of Sri Jayewardenepura 
macroinvertebrates, fish, algae and bacteria (Ramakrishna, 2003). Certain aquatic plants have also been used as indicator species to monitor pollution status and nutrient enrichment of a water body (Batiuk et $a l ., 1992)$. It has been documented that monitoring of water quality using benthic macroinvertebrates and physicochemical parameters to determine the biological richness of a stream is a successful method to assess the health of a water body (Duran, 2002). Fresh water flora and fauna play an important role as indicators of aquatic pollution as biological communities provide a more faithful reflection of environmental conditions, since they are continuously exposed and sensitive to changes in environment (Rosenberg and Resh, 1993; Tudorancea et al., 1979).

Pollution Tolerant Index (PTI) is currently used by the United States Environmental Protection Agency (USEPA) in order to determine the quality of water based on macro invertebrates. The PTI index categorizes macroinvertebrates into pollution sensitive, moderately pollution sensitive and pollution tolerant groups and are assigned with a numerical value depending on their pollution tolerance levels. Thus, there are three health status of water depending on PTI values (PTI value greater than 40, the water quality is considered as good, within 20-40 water quality is fair and the value less than 20 is considered as poor quality).

Coliform bacteria have been used to evaluate the general quality of water. Moreover, fecal coliforms, Escherichia coli, Salmonella spp, zooplankton and zoobenthos are used as water quality bioindicators as they respond quickly to environmental change and may be effective indicators of alterations in water quality (Gannon and Stemberger, 1978).

An abundance of phytoplankton is indicative of nutrient pollution (De Lange, 1994). Palmer (1959) has demonstrated that algal assemblages could be used as indicators of both clean and polluted waters and Shannon Wiener Diversity Index (SWDI) can be used to assess the quality of the water depending on the diversity of phytoplankton community. Gray (1994) has documented that changes in diversity index or any other parameters of community structure can be used to assess degree of environmental stress assuming that the change in index value is related to the intensity of pollution. Analysis of physiochemical parameters of a water body gives information on the present status of health of the water body (Buss et al., 2003; Gonçalves and Menzes, 2011). Thus, the present study was carried out in Bolgoda canal and Waga stream with an aim to assess the health status of aquatic life in both water bodies with respect to some selected physico-chemical parameters and bioindicator species.

\section{Materials and Methods}

\subsection{Study areas}

This study focuses on two types of water ways: polluted and relatively unpolluted water body in the Colombo district. Bolgoda canal $\left(6^{\circ} 50^{\prime} 0^{\prime \prime} \mathrm{N}, 79^{\circ} 53^{\prime} 0^{\prime \prime} \mathrm{E}\right)$, Attidiya which is flowing in amidst of Bellanwila-Attidiya marsh is situated on the south-eastern outskirts of Colombo (Figure 1). The depth of the canal is approximately 8 feet and the flow rate is $0.0270 \mathrm{~m} / \mathrm{s}$. Most industrial effluents, including toxic dyes and other industrial waste from approximately 20 factories in Rathmalana are discharged through this canal (CEA, 1993). Bolgoda canal was chosen for the study as this area is under human pressure that includes encroachment, domestic sewage, waste water discharges, storm water runoffs and dumping of industrial waste effluents. On the other hand Waga stream (6 $\left.6^{\circ} 54^{\prime} 22^{\prime \prime} \mathrm{N}, 80^{\circ} 8^{\prime} 7^{\prime \prime} \mathrm{E}\right)$, Maththaka (Figure 2 ) is flowing across an unurbanized area in Colombo district receiving comparatively less levels of contaminants (Manage unpublished data, Waga stream). Nevertheless, domestic discharges from nearby human settlements, effluents from rubber factory, rubber estate and tea estates are added to the stream as point and non-point source pollutants.

Three locations (1, 2 and 3) were chosen in both the Bolgoda canal and Waga stream (Figure 1 and Figure 2) representing areas under human activities and relatively undisturbed areas. In the Bolgoda canal, 
location 1 received discharges from household, location 2 was a feeding and bathing place for cattle and where location 3 was situated in an undisturbed area. In the Waga steam (Figure 2) location 1 received effluent from Rubber factory, location 2 received domestic loadings and location 3 was an undisturbed area.

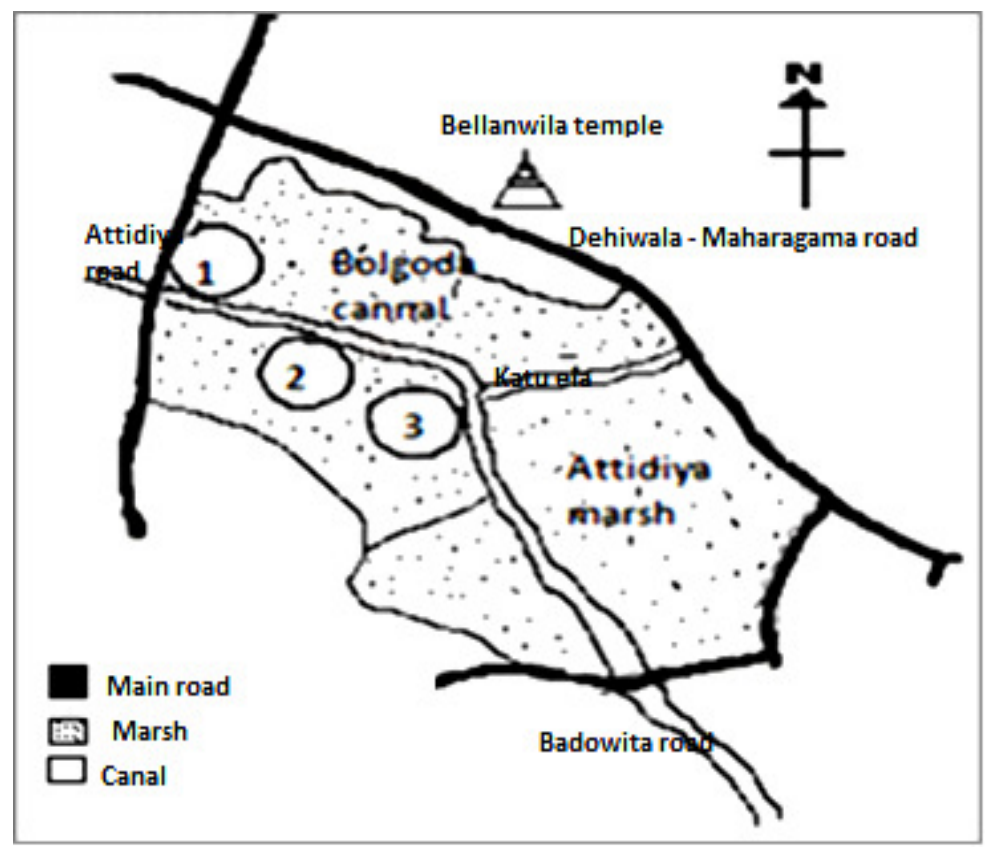

Figure 1: Sampling locations of Bolgoda canal system (Source: Wetland site report and conservation management plan, Bellanwil Attidiya marsh, 1993)

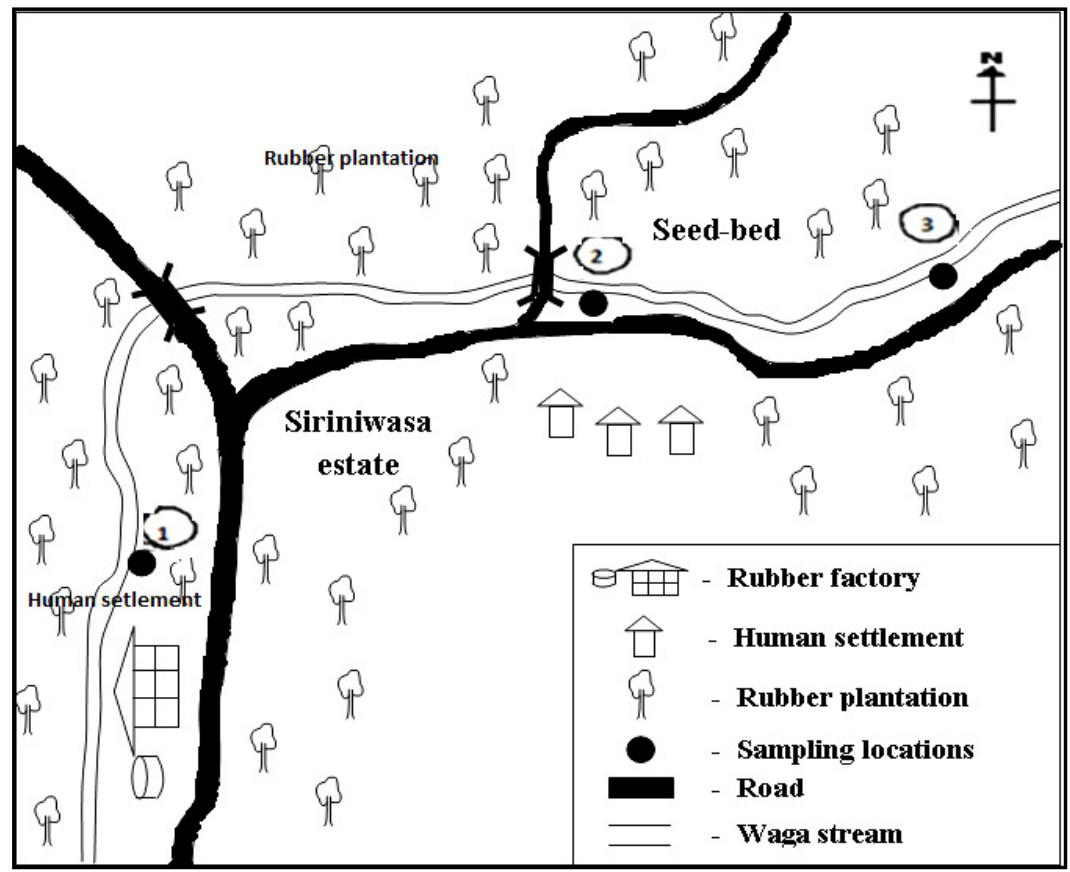

Figure 2: Sampling locations of Waga stream

\subsection{Sample collection and chemical analysis}

Monthly sampling was carried out for a period of six months from February to July 2010. Samples were collected within two days in both sites during 8.30 am to $12.00 \mathrm{pm}$. Surface water samples were collected using a $10 \mathrm{~L}$ plastic bucket and temperature $\left({ }^{\circ} \mathrm{C}\right)$, dissolved oxygen $(\mathrm{mg} / \mathrm{l}), \mathrm{pH}$ and electric 
conductivity $(\mu \mathrm{s} / \mathrm{cm})$ were measured at the site itself using thermometer (Immersion, Philip harris, England), DO meter (Oxi 320/ set, WTW Co., Weilheim, Germany), pH meter (330/ set ,WTW Co., Weilheim, Germany) and conductivity meter (340A/set,WTW Co., Weilheim, Germany) respectively. Samples were kept in an ice box during transportation and stored in $4{ }^{\circ} \mathrm{C}$ until analysis. Nitrate concentration $(\mathrm{mg} / \mathrm{l})$ and orthophosphate concentration $(\mathrm{mg} / \mathrm{l})$ were measured according to standard spectrophotometric method (Silva et al. 1996). Five days biochemical oxygen demand ( $\left.\mathrm{BOD}_{5}\right)$, alkalinity, sulphide concentration and hardness were measured within 24 hours according to standard titrometric methods.

\subsection{Biological studies}

The benthic macro invertebrate community in both water and mud was sampled in $1 \mathrm{~m}^{2}$ areas by the kicking method for 15 minutes in order to include all possible microhabitats at each station. In certain locations with the presence of large stones they were first picked out and washed into the kick net to remove larvae and other attached macroinvertebrates. In addition, macroinvertebrate samples were separated from the macrophytes and the sediment using sieves $(250 \mu \mathrm{m})$. Collected specimens were washed with same water and sorted based on morphological features and identified to the lowest possible taxon (species, genus or family). Identification was done within 4 hours of sample collection, using the standard manual of volunteer stream monitoring of United States Environmental Protection Agency (1996) and the guide for freshwater fauna of Ceylon (Mendis and Fernando, 2002). Unknown specimen samples were rinsed with distilled water and fixed with $5 \%$ formalin at the site and transferred to the laboratory for further identification. Further, identification of unknown species was carried out at the national museum, referring the available specimens and following the identification keys (Mendis and Fernando, 2002).

\subsection{Calculation of Pollution Tolerant Index (PTI)}

Pollution Tolerant Index (PTI) for the canal was calculated according to standard manual volunteer stream monitoring of USEPA.

\subsection{Analysis of phytoplankton composition}

Phytoplankton samples were collected by filtering 100 liters of water through $55 \mu \mathrm{m}$ plankton net and were fixed with acidified Lugals' solution at final concentration of $1 \%$ followed by natural sedimentation. Identification of plankton was carried out under light microscopy using standard keys (Prescott, 1970; Manage, 2012). Enumeration of phytoplankton was done using a shedwick rafter counting chamber under the light microscope (x 40).

\subsection{Calculation of Shannon Wiener Diversity Index (SWDI)}

SWDI of phytoplankton composition in the canal was calculated according to following formula.

$$
\mathrm{H}^{\prime}=-\sum_{i=1} \mathrm{p} i \ln \mathrm{p} i
$$

Where, H’: Species diversity index,

S: Number of species,

$p i$ : Proportion of individuals of each species belonging to the " $i$ " th species of the total number of individual

\subsection{Statistical analysis}

Principal component analysis (PCA) was applied using primer 5 software for physico-chemical parameters, PTI and SWDI values of Bolgoda canal and Waga stream. The effect of physico-chemical parameters on PTI and SWDI were analyzed separately by regression analysis and any significant differences were determined at a 0.05 probability level using Minitab 13.2 statistical software. 


\section{Results}

Total number of nine macroinvertebrate species belonging to three different classes were recorded (Table 1) in Bolgoda canal and they were grouped as; Gastropoda (3 species), Insecta (5 species) and Clitellata (1 species). The results showed that the Bolgoda canal possessed pollution tolerant and moderately pollution tolerant species with a range of mean PTI between $18.06 \pm 0.93$ and $19.46 \pm 0.51$ (Table 2) indicating poor quality water according to the USEPA. In contrast, total of 7 macroinvertebrate species were recorded from Waga stream; Gastropoda (2 species), Insecta (4 species) and Crustcea (1 species).

The results revealed that, the Waga stream was characterized as having pollution sensitive species with a range of mean PTI between $35.43 \pm 1.24-39.10 \pm 0.70$ (Table 4) which indicates good quality water. Among gastropods, Paludomus loricatus and Paludomus zeylanicus were recorded as local aquatic snails belonging to Sri Lanka from Waga stream and they were the dominant macroinvertebrates inhabiting throughout the stream bottom.

Table 1: Numerical occurrence of macroinvertebrates in the Bolgoda canal

\begin{tabular}{llcccccc}
\hline Class & \multicolumn{1}{c}{ Species } & February & \multicolumn{1}{c}{ March } & \multicolumn{1}{c}{ April } & May & June & July \\
\hline Gastropoda & $\begin{array}{l}\text { Planorbella } \\
\text { trivolvis }\end{array}$ & $3.00 \pm 0.50$ & $2.00 \pm 1.00$ & $2.0 \pm 0.00$ & $3.00 \pm 1.00$ & $4.00 \pm 2.00$ & $2.00 \pm 1.73$ \\
& $\begin{array}{l}\text { Promacea } \\
\text { bridgesi }\end{array}$ & $4.00 \pm 1.00$ & $5.00 \pm 2.64$ & $3.00 \pm 1.00$ & $4.00 \pm 1.00$ & $3.00 \pm 2.64$ & $5.00 \pm 2.64$ \\
& Cerithiidae sp & $12.0 \pm 3.00$ & $10.0 \pm 1.60$ & $5.00 \pm 1.73$ & $9.00 \pm 1.00$ & $13.0 \pm 3.60$ & $9.00 \pm 1.00$ \\
Insecta & Gerris sp & $3.00 \pm 1.00$ & $4.00 \pm 1.73$ & $2.00 \pm 1.00$ & $3.00 \pm 0.00$ & $3.00 \pm 1.00$ & $2.00 \pm 0.00$ \\
& $\begin{array}{l}\text { Lethocerus } \\
\text { americanus }\end{array}$ & $4.00 \pm 2.00$ & $4.00 \pm 1.00$ & $3.00 \pm 1.00$ & $2.00 \pm 1.00$ & $1.50 \pm 0.70$ & $4.00 \pm 2.64$ \\
& $\begin{array}{l}\text { Plea frontalis } \\
\text { Zygopteran }\end{array}$ & $21.0 \pm 2.60$ & $20.0 \pm 2.64$ & $13.0 \pm 2.19$ & $14.0 \pm 2.64$ & $22.0 \pm 2.07$ & $11.0 \pm 1.24$ \\
& nymphs & $4.50 \pm 3.50$ & $6.00 \pm 2.82$ & $4.00 \pm 2.64$ & $2.00 \pm 1.00$ & $3.00 \pm 1.73$ & $\mathrm{NR}$ \\
Clitellata & Nepa cinerea & $2.0 \pm 0.00$ & $\mathrm{NR}$ & $\mathrm{NR}$ & $3.00 \pm 0.00$ & $2.00 \pm 1.73$ & $1.00 \pm 0.00$ \\
& Tubifex tubifex & $1.50 \pm 2.00$ & $\mathrm{NR}$ & $2.00 \pm 1.00$ & $\mathrm{NR}$ & $1.50 \pm 0.70$ & $\mathrm{NR}$ \\
\hline
\end{tabular}

NR - Not recorded

Table 2: Pollution Tolerant Index (PTI) for indicator species in Bolgoda canal

\begin{tabular}{lcrrr}
\hline \multirow{2}{*}{ Month } & \multicolumn{4}{c}{ PTI values } \\
\cline { 2 - 4 } & \multicolumn{3}{c}{ Locations } & Mean \pm SD \\
\cline { 2 - 4 } & $\mathbf{1}$ & $\mathbf{2}$ & $\mathbf{3}$ & \\
\hline February & 19.00 & 18.60 & 19.80 & $19.13 \pm 0.61$ \\
March & 18.50 & 18.70 & 17.00 & $18.06 \pm 0.93$ \\
April & 18.90 & 19.90 & 19.80 & $19.5 \pm 0.52$ \\
May & 19.60 & 18.90 & 19.90 & $19.46 \pm 0.51$ \\
June & 18.50 & 18.90 & 18.60 & $18.66 \pm 0.21$ \\
July & 18.50 & 17.90 & 18.80 & $18.40 \pm 0.46$ \\
\hline
\end{tabular}


Table 3: Numerical occurrence of macroinvertebrates in Waga stream

\begin{tabular}{llrrrrrr}
\hline Class & Species & February & March & April & May & June & July \\
\hline Gastropoda & $\begin{array}{l}\text { Paludomus } \\
\text { loricatus } \\
\text { Paludomus } \\
\text { zeylanicus }\end{array}$ & $15 \pm 5.00$ & $10 \pm 5.00$ & $11.33 \pm 5.50$ & $9 \pm 1.73$ & $12 \pm 4.00$ & $14 \pm 4.00$ \\
Insecta & $2.3 \pm 1.50$ & $6 \pm 2.64$ & $7 \pm 1.00$ & $3 \pm 1.73$ & $4 \pm 2.64$ & $5 \pm 1.00$ \\
& $\begin{array}{l}\text { Cylindrostethus } \\
\text { produtus }\end{array}$ & $8 \pm 1.00$ & $8 \pm 2.00$ & $6.33 \pm 3.78$ & $4 \pm 1.00$ & $7 \pm 2.00$ & $6 \pm 1.00$ \\
& $\begin{array}{l}\text { Plecopteran } \\
\text { nymphs }\end{array}$ & $1.5 \pm 0.70$ & $\mathrm{NR}$ & $6 \pm 1.37$ & $\mathrm{NR}$ & $3 \pm 0.67$ & $3 \pm 1.31$ \\
& $\begin{array}{l}\text { Psephenidae } \\
\text { larvae }\end{array}$ & $8 \pm 2.00$ & $9 \pm 1.00$ & $7.66 \pm 1.50$ & $5 \pm 2.64$ & $2 \pm 1.00$ & $3 \pm 1.00$ \\
& $\begin{array}{l}\text { Ephemeropteran } \\
\text { nymphs }\end{array}$ & $1 \pm 0.02$ & $3 \pm 1.40$ & $2 \pm 1.21$ & $1 \pm 0.76$ & $1.56 \pm 0.71$ & $\mathrm{NR}$ \\
Crustacea & Aegla sp & $\mathrm{NR}$ & $3 \pm 0.52$ & $2 \pm 1.40$ & $\mathrm{NR}$ & $1 \pm 0.03$ & $\mathrm{NR}$ \\
\hline NR - not recorded
\end{tabular}

Table 4: Pollution Tolerant Index (PTI) calculated to indicator species in Waga stream

\begin{tabular}{lcccc}
\hline \multirow{2}{*}{ Month } & \multicolumn{4}{c}{ PTI values } \\
\cline { 2 - 5 } & $\mathbf{1}$ & $\mathbf{2}$ & $\mathbf{3}$ & Mean \pm SD \\
\hline February & 38.40 & 38.90 & 36.90 & $38.06 \pm 1.04$ \\
March & 36.20 & 36.10 & 34.00 & $35.43 \pm 1.24$ \\
April & 36.90 & 38.40 & 37.90 & $37.73 \pm 0.76$ \\
May & 38.40 & 38.20 & 38.20 & $38.26 \pm 0.11$ \\
June & 38.40 & 37.90 & 39.50 & $38.60 \pm 0.81$ \\
July & 38.30 & 39.40 & 39.60 & $39.10 \pm 0.70$ \\
\hline
\end{tabular}

Phytoplankton species in Bolgoda canal and Waga stream and their percentage value together with Shannon index values are given in Table 5 and Table 6. Phytoplankton composition of Bolgoda canal contained pollution tolerant algae species (Table 5) and the Shannon diversity index ranged between $0.674 \pm 0.36$ to $1.513 \pm 1.80$ during the sampling period. In Waga stream mostly clean water algae species were recorded (Table 6) and Shannon diversity index ranged between $1.89 \pm 0.72$ to $3.06 \pm 0.82$.

Table 5: Species composition and Shannon index value of phytoplankton in Bologoda canal

\begin{tabular}{|c|c|c|c|c|c|c|c|c|}
\hline \multirow{2}{*}{ Month } & \multirow{2}{*}{$\begin{array}{l}\text { Total number of } \\
\text { phytoplanktons } \\
(\text { cells } / \mathrm{ml})\end{array}$} & \multicolumn{6}{|c|}{ Percentage composition of species } & \multirow{2}{*}{$\begin{array}{c}\text { Shannon Wienner } \\
\text { index value }\end{array}$} \\
\hline & & Sp1 & $\mathrm{Sp} 2$ & Sp 3 & $\mathrm{Sp} 4$ & Sp 5 & Sp 6 & \\
\hline February & $2033 \pm 0.19$ & 45.70 & 13.40 & 7.80 & 5.90 & 21.10 & 6.10 & $0.674 \pm 0.36$ \\
\hline March & $1567 \pm 0.24$ & 34.98 & 6.78 & 3.96 & 20.07 & 28.78 & 5.43 & $1.335 \pm 0.29$ \\
\hline April & $1702 \pm 0.51$ & 40.07 & 3.90 & 2.77 & 19.71 & 25.63 & 7.92 & $1.265 \pm 0.11$ \\
\hline May & $1938 \pm 1.37$ & 42.08 & 5.32 & 2.02 & 24.22 & 23.05 & 3.31 & $1.232 \pm 1.28$ \\
\hline June & $1688 \pm 1.17$ & 36.03 & 6.71 & 3.09 & 12.46 & 29.02 & 12.51 & $1.310 \pm 0.91$ \\
\hline July & $1309 \pm 0.29$ & 25.09 & 10.31 & 5.24 & 13.43 & 28.61 & 17.32 & $1.513 \pm 1.80$ \\
\hline
\end{tabular}

Sp 1.-Spirulina sp., Sp 2.-Phacus caudatus, Sp 3.-Scenedesmus armatus, Sp 4.-Pediastrum duplex,

Sp 5-. Microcystis aeraginosa, Sp 6-.Actinastarum sp. 
Table 6: Species composition and Shannon index value of phytoplankton in Waga stream

\begin{tabular}{|c|c|c|c|c|c|c|c|}
\hline \multirow[b]{2}{*}{ Month } & \multirow{2}{*}{$\begin{array}{l}\text { Total number of } \\
\text { phytoplanktons } \\
\quad(\text { cells } / \mathrm{ml})\end{array}$} & \multicolumn{5}{|c|}{ Percentage composition of species } & \multirow{2}{*}{$\begin{array}{c}\text { Shannon wienner } \\
\text { index value }\end{array}$} \\
\hline & & Sp1 & Sp 2 & Sp 3 & Sp 4 & Sp 5 & \\
\hline February & $1024 \pm 1.31$ & 21.10 & 7.44 & 34.35 & 22.77 & 14.43 & $3.01 \pm 1.89$ \\
\hline March & $1567 \pm 0.87$ & 26.09 & 5.64 & 41.21 & 12.21 & 14.85 & $2.88 \pm 1.20$ \\
\hline April & $1011 \pm 0.51$ & 22.36 & 17.90 & 32.39 & 15.24 & 12.11 & $3.06 \pm 0.82$ \\
\hline May & $1450 \pm 1.67$ & 33.47 & 3.67 & 45.67 & 10.76 & 6.43 & $2.90 \pm 1.34$ \\
\hline June & $1788 \pm 1.82$ & 44.43 & 2.01 & 36.04 & 12.00 & 5.52 & $1.89 \pm 0.72$ \\
\hline July & $1609 \pm 0.81$ & 28.01 & 12.76 & 31.72 & 13.30 & 14.21 & $2.76 \pm 0.19$ \\
\hline
\end{tabular}

Sp 1.-Microcystis wesenbergii., Sp 2-. Ulothrix sp, Sp 3-Entzia acuta. Sp 4-Elaktothrix genevensis, Sp 5-.Staurastrum sp.

The mean water temperature of Bolgoda canal was of $29.05 \pm 0.22^{\circ} \mathrm{C}$ (Table 7) and the mean $\mathrm{pH}$ was 5.67 \pm 0.23 . The EC and DO values of the Bolgoda canal was recorded as $406.85 \pm 100.40 \mu \mathrm{s} / \mathrm{cm}$ and $3.54 \pm 0.66 \mathrm{mg} / \mathrm{l}$ respectively. Total nitrate concentration ranged between 1.22 to $1.46 \mathrm{mg} / \mathrm{l}$, while orthophosphate concentration between 4.56 to $5.45 \mathrm{mg} / \mathrm{l}$ during the study period. The mean BOD level of the Bolgoda canal was of $3.62 \pm 0.3 \mathrm{mg} / \mathrm{l}$. The mean alkalinity, sulphide and hardness concentrations were recorded as $1.69 \pm 0.19 \mathrm{~m} \mathrm{~mol} / 1,1.23 \times 10^{-4} \pm 0.27 \mathrm{~mol} / 1$, and $2.06 \pm 0.66 \mathrm{~m} \mathrm{~mol} / 1$ respectively. In Waga stream mean temperature was $25.20 \pm 1.00{ }^{\circ} \mathrm{C}$ and mean $\mathrm{pH}$ and $\mathrm{EC}$ were $6.14 \pm 0.12$ and $31.75 \pm 3.45 \mu \mathrm{s} / \mathrm{cm}$ respectively. The mean DO value was $6.99 \pm 0.75 \mathrm{mg} / \mathrm{l}$. Mean total nitrate and orthophosphate concentrations in the Waga stream were $0.63 \pm 0.25 \mathrm{mg} / 1$ and $6.18 \pm 0.78 \mathrm{mg} / 1$ respectively. Mean alkalinity value and BOD were $0.47 \pm 0.12 \mathrm{~m} \mathrm{~mol} / 1$ and $0.042 \pm 0.01 \mathrm{mg} / 1$ respectively. Sulphide and hardness were not recorded.

Table 7: Mean physico-chemical parameters in the Bologoda canal and Waga stream during the study period

\begin{tabular}{|c|c|c|c|c|}
\hline \multirow{3}{*}{$\begin{array}{c}\text { Physico-chemical } \\
\text { parameter }\end{array}$} & \multicolumn{4}{|c|}{ Water body } \\
\hline & \multicolumn{2}{|c|}{ Bolgoda canal } & \multicolumn{2}{|c|}{ Waga stream } \\
\hline & Range & Mean & Range & Mean \\
\hline Temperature $\left({ }^{\circ} \mathrm{C}\right)$ & $28.30-29.80$ & $29.05 \pm 0.22$ & $24.20-26.20$ & $25.20 \pm 1.00$ \\
\hline $\mathrm{pH}$ & $5.45-5.90$ & $5.67 \pm 0.23$ & $6.02-6.25$ & $6.14 \pm 0.12$ \\
\hline Electric conductivity $(\mu \mathrm{s} / \mathrm{cm})$ & $306.40-507.30$ & $406.85 \pm 100.40$ & $28.30-35.20$ & $31.75 \pm 3.45$ \\
\hline $\mathrm{DO}(\mathrm{mg} / \mathrm{l})$ & $2.88-4.20$ & $3.54 \pm 0.66$ & $6.80-7.30$ & $6.99 \pm 0.75$ \\
\hline Total nitrate (mg/l) & $1.22-1.46$ & $1.35 \pm 0.09$ & $0.32-0.97$ & $0.63 \pm 0.25$ \\
\hline Orthosphate (mg/l) & $4.56-5.45$ & $5.14 \pm 0.5$ & $5.30-6.80$ & $6.18 \pm 0.78$ \\
\hline BOD (mg/l) & $3.26-3.89$ & $3.62 \pm 0.3$ & $0.032-0.054$ & $0.042 \pm 0.01$ \\
\hline Alkalinity (m mol/l) & $1.51-1.90$ & $1.69 \pm 0.19$ & $0.35-0.60$ & $0.47 \pm 0.12$ \\
\hline Sulphide $\left(10^{-4} \mathrm{~mol} / \mathrm{l}\right)$ & $0.92-1.42$ & $1.23 \pm 0.27$ & NR & NR \\
\hline Hardness( $\mathrm{m} \mathrm{mol} / \mathrm{l})$ & $1.30-2.50$ & $2.06 \pm 0.66$ & NR & NR \\
\hline
\end{tabular}

NR-Not recorded

The principal component analysis (PCA) produced two principal components that collectively explained $100.0 \%$ of the variance of physico-chemical parameters, pollution tolerant index and Shannon diversity index in Bolgoda canal and Waga stream (Table 8 and 9). PCA ordination of the reaches according to the 12 variables describing physico-chemical parameters, pollution tolerant index and Shannon diversity index provided a strong discrimination of the three sites in Bolgoda canal and Waga stream (Figures 3 and 4). 
Table 8: Axis eigenvalues and weighted correlations between physico-chemical parameters, PTI and SWDI variables of PCA of Bolgoda canal samples variables that explain a significant amount of variation amongst samples following forward selection $(* p<0.05)$.

\begin{tabular}{lrr}
\hline Variable & PC1 & PC2 \\
\hline Eigen value & 6.71 & 5.29 \\
\% of Var. & 55.9 & 44.1 \\
Cumulative value & 55.9 & 100.0 \\
PTI & -0.140 & 0.405 \\
SWDI & -0.374 & 0.105 \\
Temperature $\left({ }^{\circ} \mathrm{C}\right)$ & 0.307 & -0.264 \\
pH & 0.386 & 0.002 \\
Electric conductivity $(\mu \mathrm{s} / \mathrm{cm})$ & -0.219 & -0.358 \\
DO $(\mathrm{mg} / \mathrm{l})$ & -0.245 & -0.336 \\
Total nitrate $(\mathrm{mg} / \mathrm{l})$ & -0.368 & 0.132 \\
Orthophosphate $(\mathrm{mg} / \mathrm{l})$ & 0.201 & -0.371 \\
BOD $(\mathrm{mg} / \mathrm{l})$ & -0.372 & -0.115 \\
Alkalinity $(\mathrm{m} \mathrm{mol} / \mathrm{l})$ & -0.038 & -0.433 \\
Sulphide $\left(\mathrm{X} 10^{-4} \mathrm{~mol} / \mathrm{l}\right)$ & 0.160 & 0.396 \\
Hardness $(\mathrm{m} \mathrm{mol} / \mathrm{l})$ & -0.384 & -0.042 \\
\hline
\end{tabular}

Table 9: Axis Eigenvalues and weighted correlations between physico-chemical parameters, PTI and SWDI variables of PCA of Waga stream samples variables that explain a significant amount of variation amongst samples following forward selection $\left({ }^{*} p<0.05\right)$.

\begin{tabular}{lrr}
\hline \multicolumn{1}{c}{ Variable } & PC1 & \multicolumn{1}{c}{ PC2 } \\
\hline Eigen value & 8.49 & 1.51 \\
\% of Var. & 84.9 & 15.1 \\
Cum \% & 84.9 & 100.0 \\
PTI & -0.294 & 0.419 \\
SWDI & -0.327 & -0.246 \\
Temperature $\left({ }^{\circ} \mathrm{C}\right)$ & -0.260 & -0.530 \\
pH & 0.253 & 0.550 \\
Electric conductivity $(\mu \mathrm{s} / \mathrm{cm})$ & 0.332 & -0.206 \\
DO(mg/l) & -0.343 & 0.044 \\
Total nitrate $(\mathrm{mg} / \mathrm{l})$ & 0.343 & 0.028 \\
Orthophosphate $(\mathrm{mg} / \mathrm{l})$ & 0.343 & 0.015 \\
BOD $(\mathrm{mg} / \mathrm{l})$ & 0.318 & -0.306 \\
Alkalinity $(\mathrm{m} \mathrm{mol} / \mathrm{l})$ & 0.332 & -0.207 \\
\hline
\end{tabular}

The PCA plot of Bolgoda canal (Figure 3) confirmed that site 1 is characterized by high temperatures and $\mathrm{pH}$, moderate orthophosphate concentrations, EC and alkalinity values. Site 2 was characterized by high, nitrate concentrations, orthophosphate concentrations, EC, BOD, alkalinity and high SWDI values. Further, these characteristics were shared by site 3 with high nitrate, BOD and high SWDI values but comparatively high PTI values than the other two sites. 


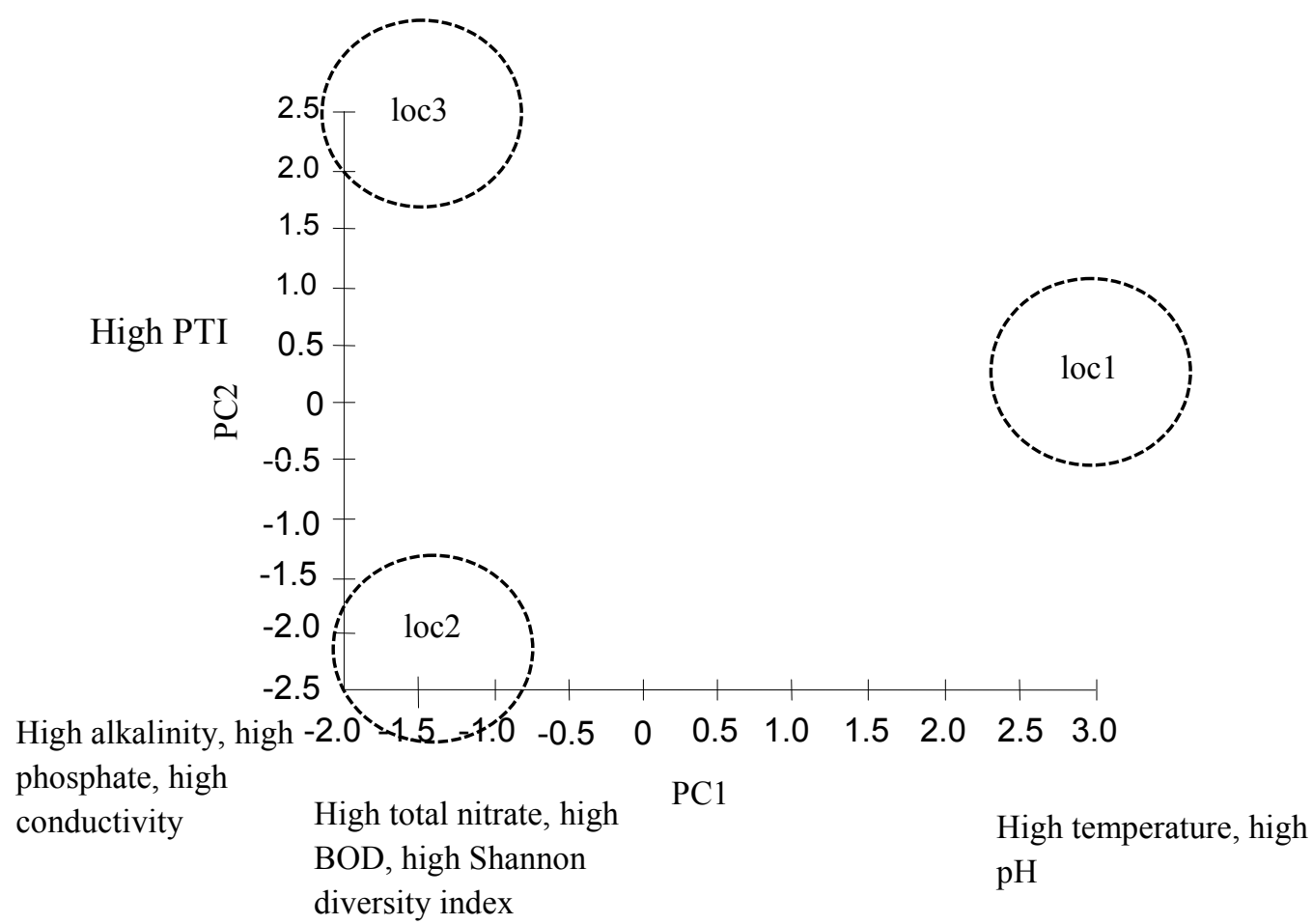

Figure 3: Ordination of the three study sites based on $\mathrm{PC}_{1}$ and $\mathrm{PC}_{2}$ scores of Principal Component Analysis between physico-chemical parameters, PTI and SWDI among the three sites of Bolgoda canal

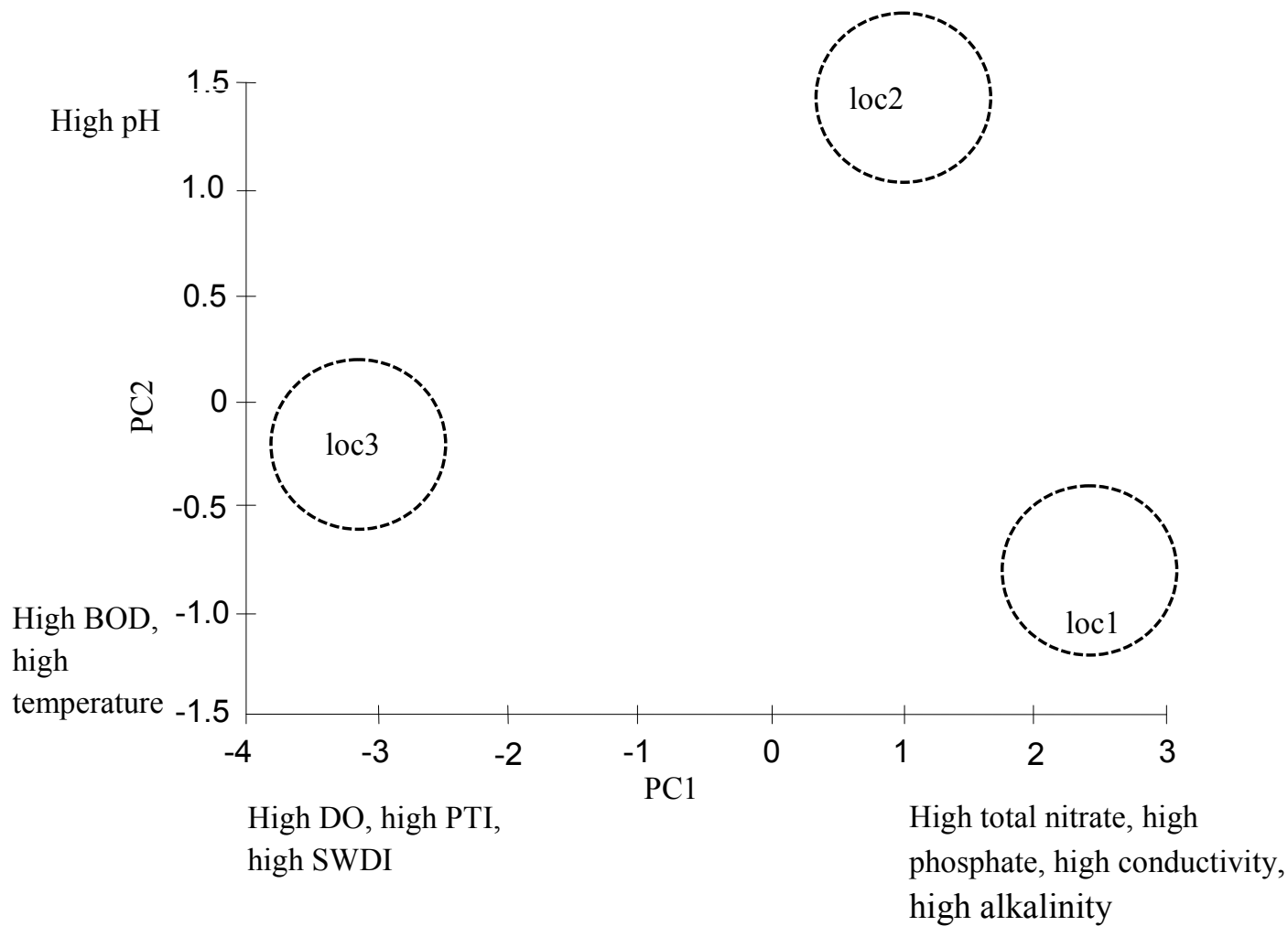

Figure 4: Ordination of the three study sites based on $\mathrm{PC}_{1}$ and $\mathrm{PC}_{2}$ scores of Principal Component Analysis between physico-chemical parameters, PTI and SWDI among the three sites of Waga stream. 
The PCA plot for Waga stream (Figure 4) indicated high BOD, temperature, nitrate, orthophosphate, EC and alkalinity in site 1. Site 2 shared some of the similar values from site 1 like high nitrate, orthophosphate, EC and alkalinity but with comparatively high $\mathrm{pH}$ value. Site 3 was characterized by high BOD, temperature, DO, PTI and high SWDI values. High cumulative percentage as high as $100.0 \%$ of the total variation of the physico-chemical parameters PTI and SWDI could be explained by both $\mathrm{PC}_{1}$ and $\mathrm{PC}_{2}$ axis.

The regression analysis was made between physico-chemical parameters and PTI values of Bolgoda canal and Waga stream are presented in Table 10. The results convinced temperature, $\mathrm{pH}, \mathrm{DO}$, nitrate and BOD had a significant effect on the PTI value of the Bolgoda canal and Waga stream. Regression analysis between physico-chemical parameters and SWDI of theBolgoda canal and Waga stream (Table 11) showed temperature, $\mathrm{pH}, \mathrm{DO}$, nitrate concentration and BOD values had a significant effect on SWDI values of Bolgoda canal at $p<0.05$ significance level and temperature, $\mathrm{pH}, \mathrm{DO}$, nitrate and orthophosphate had a significant effect at $p<0.05$ significance level on the SWDI of Waga stream.

Table 10: Regression values between the physico-chemical parameters and PTI of Bolgoda canal and Waga stream at significance level $p<0.05$

\begin{tabular}{lrr}
\hline Parameter & $\begin{array}{c}\text { Bolgoda canal } \\
(p \text { value })\end{array}$ & $\begin{array}{r}\text { Waga stream } \\
(p \text { value })\end{array}$ \\
\hline Temperature $\left({ }^{\circ} \mathrm{C}\right)$ & 0.038 & 0.046 \\
$\mathrm{pH}$ & 0.003 & 0.01 \\
Electric conductivity $(\mu \mathrm{s} / \mathrm{cm})$ & 0.214 & 0.108 \\
DO $(\mathrm{mg} / \mathrm{l})$ & 0.003 & 0.001 \\
Total nitrate $(\mathrm{mg} / \mathrm{l})$ & 0.001 & 0.003 \\
Orthophosphate $(\mathrm{mg} / \mathrm{l})$ & 0.362 & 0.582 \\
BOD $(\mathrm{mg} / \mathrm{l})$ & 0.04 & 0.006 \\
Alkalinity $(\mathrm{m} \mathrm{mol} / \mathrm{l})$ & 0.456 & 0.882 \\
Sulphide $\left(\mathrm{x} \mathrm{10} 0^{-4} \mathrm{~mol} / \mathrm{l}\right)$ & 0.679 & $\mathrm{NR}$ \\
Hardness $(\mathrm{m} \mathrm{mol} / \mathrm{l})$ & 0.061 & $\mathrm{NR}$ \\
\hline
\end{tabular}

NR-Not recorded

Table.11. Regression values between the physico-chemical parameters and SWDI of Bolgodacanl and Waga stream at significance level $p<0.05$

\begin{tabular}{lrr}
\hline \multicolumn{1}{c}{ Parameter } & $\begin{array}{c}\text { Bolgoda canal } \\
(p \text { value })\end{array}$ & $\begin{array}{c}\text { Waga stream } \\
(p \text { value })\end{array}$ \\
\hline Temperature $\left({ }^{\circ} \mathrm{C}\right)$ & 0.022 & 0.026 \\
$\mathrm{pH}$ & 0.028 & 0.042 \\
Electric conductivity $(\mu \mathrm{s} / \mathrm{cm})$ & 0.401 & 0.361 \\
DO $(\mathrm{mg} / \mathrm{l})$ & 0.0330 & 0.031 \\
Total nitrate $(\mathrm{mg} / \mathrm{l})$ & 0.0429 & 0.004 \\
Orthophosphate $(\mathrm{mg} / \mathrm{l})$ & 0.914 & 0.001 \\
BOD $(\mathrm{mg} / \mathrm{l})$ & 0.021 & 0.518 \\
Alkalinity $(\mathrm{m} \mathrm{mol} / \mathrm{l})$ & 0.128 & 0.109 \\
Sulphide $\left(\mathrm{x} 10^{-4} \mathrm{~mol} / \mathrm{l}\right)$ & 0.381 & $\mathrm{NR}$ \\
Hardness $(\mathrm{m} \mathrm{mol} / \mathrm{l})$ & 0.273 & $\mathrm{NR}$ \\
\hline
\end{tabular}

NR - Not recorded 


\section{Discussion}

Aquatic communities are good indicators of environmental quality (Buss et al. 2003). Results of Bolgoda canal also convinces this fact as most of the organisms found in the canal were moderately pollution tolerant or pollution tolerant organisms (Table 1). Especially Promacea bridgesi (apple snail) found in the canal reflects the quality of the stream as Promacea bridgesi is an invasive pouch snail living in polluted environments and generally indicate nutrient enriched conditions and poor water quality. The lung and gill combination of the Promacea bridgesi reflects their adaptation to habitats with oxygen poor water (Brown, 2009).

In the Bolgoda canal Nepa cinerea (water scorpions) was detected in sampling location 1 and 2 . It has been documented that water scorpions can tolerate pollution for a certain range (Brown, 2009) and is commonly found lurking in trash and mud in ponds and mellow, marshy streams. Presence of water scorpion in the Bolgoda canal is an indication of presence of moderately pollution tolerant species. Presence of Tubifex tubifex in the Bolgoda canal water is also ideal indications of unclean water. Kazanci, (1996) recorded that Tubifex tubifex was found in the regions in which the organic pollution was high and the dissolved oxygen was low. Thus, presence of Tubifex tubifex is compatible with low values of DO in Bolgoda canal waters (Table 9).

The macroinvertebrates in Waga stream (Table 3) consists of Psephenidae larvae, nymphs of Plecopterans and Ephimeropterans, and gilled snails like Paludomous loricatus and Paludomous zeylanicus which are categorized as pollution sensitive organisms (USEPA, 1996). The presence of pollution sensitive bioindicators in Waga stream indicates that the stream water is in good condition.

According to the USEPA, 1996 PTI value of the Bolgoda canal (Table 2) was less than 20 indicating poor water qualities where PTI value in Waga stream (Table 4) was 20-40 and further ensures fair water quality.

The phytoplankton communities recorded in the Bolgoda canal (Table 5) during the study period were mostly pollution tolerant species. Microcystis aeruginosa was the dominant algae in the canal during the study period and the presence of the Actinatarum sp. indicates polluted status of water (Manage, 2009). The Shannon Wienner Diversity Index (SWDI) value is said to be greater than 3 in clean waters, ranging from 1 to 3 indicate moderately polluted waters and less than 1 in heavily polluted waters (Trivedi, 1981).In the present study, mean SWDI value of phytoplankton species in the Bolgoda canal ranged between $0.674 \pm 0.36$ to $1.513 \pm 1.80$ indicating heavily polluted statuses. From February to July 2010 total phytoplankton counts reduced from $2033 \pm 0.19$ cells ml $^{-1}$ to $1309 \pm 0.29$ cells ml ${ }^{-1}$ (Table 5) was accompanied by increase of mean SWDI value from $0.674 \pm 0.36$ to $1.513 \pm 1.8$ respectively. It was detected that decrease of the dominant $M$. aeruginosa results in a higher SWDI value, which shows that diversity of the phytoplankton community in the Bolgoda canal is inversely proportional to density of $M$. aeruginosa.

The Waga stream has a community of clean water algae such as Staurastrum sp, Ulothrix sp, Elaktothrix zonata (Manage, 2009). The total phytoplankton numbers were found to vary from $1011 \pm 0.51$ cells $\mathrm{ml}^{-1}$ to $1788 \pm 1.82$ cells $\mathrm{ml}^{-1}$. Reduction in total counts is accompanied by increase in mean SWDI value from $1.89 \pm 0.72$ to $3.06 \pm 0.82$ indicating the stream has a diverse phytoplankton community which confirms good water quality (Table 6).

According to ambient water quality standards for inland waters in Sri Lanka, published by the Central Environmental Authority in 2001 the $\mathrm{pH}$ of the water should be 6.0 to 8.5 to sustain healthy aquatic life. The Bolgoda canal waters had much less $\mathrm{pH}$ values and deviates from maintaining healthy aquatic life. Favorable DO levels for aquatic life should be at least $3 \mathrm{mg} / \mathrm{l}$ and in the Bolgoda canal, DO decreased to $2.88 \mathrm{mg} / \mathrm{l}$ (Table 9) in February. The EC values and temperature of the Bolgoda canal waters did not exhibit a great threat to aquatic life. 
Results of the Waga stream imply that the water quality parameters of the stream was suitable to maintain a healthy aquatic life, where $\mathrm{pH}$ of the stream ranged between 6.02-6.25, EC ranged between $31.75 \pm 3.45$ and DO levels $6.99 \pm 0.75 \mathrm{mg} / 1$ showed suitability for healthy aquatic life.

PCA plot resulted classifying three sampling locations in the Bolgoda canal according to their physico-chemical parameters, PTI and SWDI (Figure 3). Location 1 in Bolgoda canal had high values of water temperature and $\mathrm{pH}$ with low PTI and SWDI. Receiving of house hold waste and anthropogenic loading by location 1 is believed to have resulted in high temperature and $\mathrm{pH}$ and low macroinvertebrate and phytoplankton diversity. The second sampling location of the canal had high total nitrate, BOD, alkalinity, orthophosphate concentration, EC and SWDI values as the location 2 was used for cattle bathing and other animal husbandry activities and consequently received high nitrates and phosphates. Thus, the location 2 was enriched with nutrients needed for algal growth which resulted in higher SWDI values. In the present study location 3 was selected as a control site and the PCA plot showed high total nitrate, high BOD values together with high PTI and SWDI indicating the site is comparatively able to maintain a diverse group of pollution tolerant macroinvertebrates and polluted water phytoplankton community.

PCA ordination of the reaches according the 12 variables describing physico-chemical parameters, PTI and SWDI provided a strong discrimination of the three distinct groups in Waga stream (Figure 4). High temperature, conductivity, total nitrate, orthophosphate, BOD, and alkalinity, in the location 1, is believed to have resulted due to effluents reaching from rubber factory resulting low PTI and SWDI values. Location 2 showed similar physico-chemical parameter values to location 1, though high $\mathrm{pH}$, low BOD and temperature. Household effluents reaching to the location 2 is believed to have resulted in high nitrate, orthophosphate, EC, and alkalinity values, though the BOD levels were much less even without receiving much organic wastes in comparison to location 1 . The third sampling location (location 3 ) of Waga stream acted as a control site and the results of PCA further convince this fact as high temperature and DO values, with a high PTI and SWDI values were recorded in location 3. Location 3 was able to maintain a diverse group of pollution sensitive macroinvertebrate group and clean water phytoplankton community in higher numbers as the high DO levels in water favors the existence of pollution sensitive species. Though, high BOD values recorded in location 3 does not agree with other parameter values and organic wastes resulted by decay of macroinvertebrates in location 3 is believed to have resulted in this condition.

Regression analysis showed among the measured physico-chemical parameters, temperature, $\mathrm{pH}$, DO, nitrate concentration and BOD had a significant positive effect on the PTI value of the Bolgoda canal and Waga stream with a $r^{2}>50 \%$ and significance level $\mathrm{p}<0.05$ (Table 10). Thus, these parameters can affect the abundance and distribution of pollution tolerant species and pollution sensitive species in Bolgoda canal and Waga stream respectively. Regression analysis between physico-chemical parameters and SWDI of Bolgoda canal and Waga stream (Table 11) showed temperature, $\mathrm{pH}, \mathrm{DO}$, nitrate and BOD values had a significant effect on SWDI values of Bolgoda canal with a $\mathrm{r}^{2}>50 \%$ and significance level $\mathrm{p}<0.05$. Bolgoda canal contained phytoplankton species which are dominant in polluted waters and had a low SWDI value. Thus, low $\mathrm{pH}, \mathrm{DO}$ and high nitrate loadings to the canal has reached for a state of eutrophication with a SWDI less than 1 in certain months. In Waga stream temperature, $\mathrm{pH}, \mathrm{DO}$, nitrate concentration and orthophosphate concentrations had a significant effect with an $\mathrm{r}^{2}>50 \%$ and significance level $\mathrm{p}<0.05$ on the SWDI.SWDI values of Waga stream ranged between 1.89 and 3.01 . SWDI $>3$ represents clean waters (Trivedi, 1981) thus, the Waga stream is a not a polluted water body.

Thus, the present study convinces that the physico-chemical parameters of Bolgoda canal water are not up to the standard level to maintain healthy aquatic life. The poor quality of the water in the canal is suitable only for moderately pollution tolerant and pollution tolerant organisms where as Waga stream owns good quality waters to sustain pollution sensitive organisms. 


\section{Conclusion}

The physico-chemical parameters like temperature, $\mathrm{pH}, \mathrm{DO}$, nitrate concentration, $\mathrm{BOD}$ and orthophosphate concentrations of a water body have a significant effect on the PTI and SWDI value. PTI, SWDI and physico-chemical parameters of Bolgoda canal and Waga stream convinced that the Bolgoda canal is suitable for pollution tolerant and moderately pollution tolerant organisms while Waga stream waters are suitable for pollution sensitive organisms. Thus, in the prediction of the health status of a water way it is important to analyze the biological component together with physico-chemical parameters as the physico-chemical parameters have a significant effect on the biological component of a water way.

\section{References}

Batiuk, R.A., Orth, R.J., Moore, K.A., Dennison, W.C., Stevension, J.C., Staver, L.W., Carter, V., Rybicki, N.B., Hickman, R.E., Kollar,S., Bieber,S., and Heasly ,P. 1992. Chesapeake Bay Submerged Aquatic Vegetation Habitat Requirements and Restoration Targets : A Technical Synthesis. Virginia Inst. of Marine Science., Gloucester Point VA United states, 258.

Brown, D., and Czarneki , J , 2009.Biological monitoring, Introduction to Volunteer Water Quality Monitoring Training Notebook, Missouri stream team, Jefferson City, United States ,140-159.

Buss, D.F., Baptista, D.F., Silveira, M.P., Nessimian, J.L ., and Dorville, L.F.M. 2003. Influence of water chemistry and Environmental quality on the macroinvertebrate assemblages in a river Basin in southeast Brazil: Hydrobiologia 481:125-136.

Central Environmental Authority, 2001. Proposed Ambient Water quality Standards for Inland Waters Sri Lanka. Colombo, Sri Lanka: Environment Action 1 Project (Funded by ADB).

De Lange, E. 1994. Manual for Simple Water Quality Analysis.International Water Tribunal (IWT) Foundation : Amsterdam.

Duran M., 2002.Monitoring water quality using benthic macroinvertebrates and physic-chemical parameters of Bezhat stream, Turkey: journal of environmental pollution 15, 709-717.

Gannon, J. E., and Stemberger ,R. S., 1978. Zooplankton as indicators of water quality.Trans. Am.Microscop. Soc. 97, 16-35.

Gonçalves, B.F., and De Menzes ,M.S., 2011. a comparative analysis of biotic indices that use macroinvetbrates to asses water quality in a coastal river of parana estate, southern brazil: Biota neotrop 11, pg 4-5.

Gray N.F., 1994.Water Technology-An introduction for environmental scientist and engineers , Viva Books Private ltd ,New Delhi, 133-177

Kazanci , N., and Girgin , S.,1996. Distribution of Oligochaeta species as bioindicators of organic pollution in Ankara Stream and their use in biomonitoring, turkey

Kennish, M.J., 1992. Ecology of Estuaries : anthropogenic effects. CRC Press : Boca Raton.

Manage, P.M., 2009. Training manual on role of Nuisance algae and their detection in water supply projects in Sri lanka, National Water supply and drainage board, Sri lanka.

McDonald, B., Borden, and W., Lathrop, J., 1990. Citizen Stream Monitoring: A Manual for Illinois. Illinois Department of Energy and Natural Resources,ILENR/RE-WR-90/18. Springfield, Illinois.

Mendis, A.S.., and Fernando, C.H., 2002.A guide to the freshwater fauna of Ceylon. In (Eds: Fernando C.H. and Weerewardhena SR) Sri Lanka Freshwater fauna and fisheries. 51-59.

Palmer, C.M., 1959. Algae in water suppliesUS Dept. of Health, pub Health service publications, Cincinnati, The Ohio journal of science, 27.

Presscott, G. W., 1970. How to know the fresh water algae, Library of congress catalog in data, 2-89.

Ramakrishnan, N., 2003.Bio-monitoring approaches for water quality assessment in two Waterbodies At tiruvannamalai, tamilnaduindia, Proceedings of the Third International Conference on Environment and Health, Chennai, India, $374-385$. 
Rosenberg, D.M., and Resh,V.H., 1993.Freshwater biomonitoring and benthic macroinvertebrates, Chapman \& Hall, New York.

Silva , E.I.L.,Namarathne ,S.Y., Weerasooriya ,S.V.R., and Munaweera , L.,1996.Water analysis user friendly field / laboratory manual, Aj prints, Dehiwala, Sri lanaka.

Trivedi R.C., 1981. Use of diversity index in evaluation of water quality, Central board for the prevention and control of water pollution, New Delhi.

Tudorancea, C., Green, R.H., Huebner, J.,1979. Structure, dynamics and production of the benthic fauna in lake Manitoba. Hydrobiologia 64(1): 59-95.

United states environmental protection agency, volunteer stream manual: A methods manual,1996

Wetland site report and conservation management plan, Bellanwila-Attidiya marsh. 1993. 1-88. 